Article

\title{
The Diversity of Bacteria Associated with the Invasive Gall Wasp Dryocosmus kuriphilus, Its Galls and a Specialist Parasitoid on Chestnuts
}

\author{
Xiaohui Yang ${ }^{1, *} *$, Yu Hui ${ }^{1}$, Daohong Zhu ${ }^{2}{ }^{\circ}$, Yang Zeng ${ }^{2}$, Lvquan Zhao ${ }^{3}$, Xuemei Yang ${ }^{1}$ and Yumei Wang ${ }^{1}$ \\ 1 College of Life Science, Hunan Normal University, Changsha 410081, China; yuhui@hunnu.edu.cn (Y.H.); \\ xuemeiyang@hunnu.edu.cn (X.Y.); wym@hunnu.edu.cn (Y.W.) \\ 2 Laboratory of Insect Behavior and Evolutionary Ecology, Central South University of Forestry and Technology, \\ Changsha 410004, China; dhzhu@csuft.edu.cn (D.Z.); t20162281@csuft.edu.cn (Y.Z.) \\ 3 Co-Innovation Center for Sustainable Forestry in Southern China, College of Forestry, \\ Nanjing Forestry University, Nanjing 210037, China; zhaolvquan@njfu.edu.cn \\ * Correspondence: xhyang@hunnu.edu.cn
}

check for

updates

Citation: Yang, X.; Hui, Y.; Zhu, D.; Zeng, Y.; Zhao, L.; Yang, X.; Wang, Y. The Diversity of Bacteria Associated with the Invasive Gall Wasp Dryocosmus kuriphilus, Its Galls and a Specialist Parasitoid on Chestnuts. Insects 2022, 13, 86. https:/ / doi.org/ $10.3390 /$ insects 13010086

Academic Editor: Mark Fellowes

Received: 30 November 2021

Accepted: 10 January 2022

Published: 13 January 2022

Publisher's Note: MDPI stays neutral with regard to jurisdictional claims in published maps and institutional affiliations.

Copyright: (C) 2022 by the authors. Licensee MDPI, Basel, Switzerland. This article is an open access article distributed under the terms and conditions of the Creative Commons Attribution (CC BY) license (https:// creativecommons.org/licenses/by/ $4.0 /)$
Simple Summary: The insect Dryocosmus kuriphilus induces galls on chestnut trees. Torymus sinensis is a host-specific parasitoid of $D$. kuriphilus and phenologically synchronizes with $D$. kuriphilus. The aim of this research is to investigate the bacterial communities and predominant bacteria of D. kuriphilus, T. sinensis, D. kuriphilus galls and the galled twigs of Castanea mollissima. We provide the first evidence that D. kuriphilus shares most bacterial species with T. sinensis, D. kuriphilus galls and galled twigs. The predominant bacteria of D. kuriphilus are Serratia sp. and Pseudomonas sp. Many species of the Serratia and Pseudomonas genera are plant pathogenic bacteria, and we suggest that D. kuriphilus may be a potential vector of plant pathogens. Furthermore, a total of 111 bacteria are common to D. kuriphilus adults, T. sinensis, D. kuriphilus galls and galled twigs, and we suggest that the bacteria may transmit horizontally among $D$. kuriphilus, $T$. sinensis, D. kuriphilus galls and galled twigs on the basis of their ecological associations.

Abstract: Dryocosmus kuriphilus (Hymenoptera: Cynipidae) induces galls on chestnut trees, which results in massive yield losses worldwide. Torymus sinensis (Hymenoptera: Torymidae) is a hostspecific parasitoid that phenologically synchronizes with $D$. kuriphilus. Bacteria play important roles in the life cycle of galling insects. The aim of this research is to investigate the bacterial communities and predominant bacteria of D. kuriphilus, T. sinensis, D. kuriphilus galls and the galled twigs of Castanea mollissima. We sequenced the V5-V7 region of the bacterial 16S ribosomal RNA in D. kuriphilus, T. sinensis, D. kuriphilus galls and galled twigs using high-throughput sequencing for the first time. We provide the first evidence that $D$. kuriphilus shares most bacterial species with T. sinensis, D. kuriphilus galls and galled twigs. The predominant bacteria of D. kuriphilus are Serratia sp. and Pseudomonas sp. Furthermore, the bacterial community structures of D. kuriphilus and T. sinensis clearly differ from those of the other groups. Many species of the Serratia and Pseudomonas genera are plant pathogenic bacteria, and we suggest that $D$. kuriphilus may be a potential vector of plant pathogens. Furthermore, a total of 111 bacteria are common to D. kuriphilus adults, T. sinensis, D. kuriphilus galls and galled twigs, and we suggest that the bacteria may transmit horizontally among D. kuriphilus, T. sinensis, D. kuriphilus galls and galled twigs on the basis of their ecological associations.

Keywords: Dryocosmus kuriphilus; Torymus sinensis; diversity; bacterial community; galls; highthroughput sequencing; Castanea mollissima

\section{Introduction}

Plant galls are outgrowths of plant tissues induced by a wide variety of organisms, including protists, nematodes, mites, fungi, bacteria and insects [1]. Galling insects manipulate the development of host plants and induce galls on different organs of the host 
plants [2]. Galling insects are a highly sophisticated herbivore group, including gall wasps, gall midges, gall aphids, gall moths, thrips and psyllids [3].

The gall wasp Dryocosmus kuriphilus (Hymenoptera: Cynipidae) is native to China and has rapidly spread throughout Asia, Europe and North America [4,5]. D. kuriphilus is a univoltine and parthenogenetic species that lays eggs in the buds of Castanea spp. during the summer, and their larvae overwinter inside the buds and induce galls on the host plants during the following spring [6]. Galls are the sole food sources for D. kuriphilus [7,8]. Torymus sinensis (Hymenoptera: Torymidae) is a univoltine and host-specific parasitoid that phenologically synchronizes with D. kuriphilus [9]. During April and May, the female adults of T. sinensis lay eggs in newly formed galls or on the bodies of D. kuriphilus larvae $[10,11]$. After hatching, the larvae of T. sinensis feed ectoparasitically on the mature larvae of $D$. kuriphilus. In the winter, the female adults of T. sinensis overwinter inside D. kuriphilus galls [11]. In the following spring, the T. sinensis adults emerge from the overwintering withered galls that were induced by D. kuriphilus the previous year [12,13]. Thus, the ecological interactions among D. kuriphilus, T. sinensis, D. kuriphilus galls and galled twigs include oviposition, gall formation, parasitism and feeding [14].

Bacteria play important roles in the life cycle of galling insects [15]. For example, Microbacterium sp. of Leptocybe invasa can resist the secondary metabolites of plants and expand the range of host feeding, which is beneficial for the colonization of $L$. invasa $[16,17]$. Buchnera aphidicola synthesizes essential amino acids for gall aphids and facilitates the stress tolerance and reproduction of the host $[18,19]$. Furthermore, Wolbachia may cause the parthenogenesis of some galling insects [20]. However, previous studies have indicated that thelytokous parthenogenesis of D. kuriphilus may not be associated with Wolbachia [21] To date, no studies have reported the effects of bacteria on $D$. kuriphilus and T. sinensis.

Bacteria infect D. kuriphilus, T. sinensis and galls. Previous studies have isolated Staphylococcus saprophyticus, Paenibacillus sp., Paenibacillus sp. and Pseudomonas fluorescens from the body of D. kuriphilus [22]. Notably, the species of Pseudomonas genus are the predominant bacteria in many galling insects, including gall midges, gall aphids, gall sawflies, thrips and L. invasa $[17,18,23-27]$. Furthermore, $T$. sinensis are infected with the symbiotic bacteria Wolbachia [28]. For cynipid galls, high-throughput sequencing analyses have confirmed that the predominant endophytic bacteria of Lithosaphonecrus arcoverticus (Hymenoptera: Cynipidae) galls are Allorhizobium, Chryseobacterium, Curtobacterium, Luteibacter, Pantoea and Stenotrophomonas genera [29]. However, the difference between the bacterial communities of D. kuriphilus, T. sinensis and D. kuriphilus galls and galled twigs within the same habitat remains unclear. Furthermore, some galling insects may acquire bacteria from host plants [23-26], and it remains unclear whether D. kuriphilus, T. sinensis and D. kuriphilus galls acquire subsets of the bacteria present in the galled twigs

In this study, the bacteria within the body of D. kuriphilus, T. sinensis, D. kuriphilus galls and the galled twigs of Castanea mollissima were detected by high-throughput sequencing for the first time in order to explore the potential horizontal transmission of bacteria among D. kuriphilus, T. sinensis, D. kuriphilus galls and galled twigs. The $\alpha$-diversity, community structures and predominant species of bacteria associated with D. kuriphilus, T. sinensis, D. kuriphilus galls and galled twigs were compared at the species level, which is the level of taxonomic classification of bacteria. We discuss horizontal transmission, differences in community structure and the predominant species of bacteria in D. kuriphilus, T. sinensis, D. kuriphilus galls and galled twigs on the basis of their ecological associations.

\section{Materials and Methods}

\subsection{Collection and Pretreatment of Specimens}

D. kuriphilus galls, the galled twigs of $C$. mollissima and overwintering withered galls were collected in Huangqiao Town $\left(27.02^{\circ} \mathrm{N} / 110.85^{\circ} \mathrm{E}\right)$, China, from April to May 2019. The D. kuriphilus galls grow and develop on the galled twigs (Figure S1). The overwintering withered gall refers to $D$. kuriphilus galls induced by D. kuriphilus in the previous year. One D. kuriphilus gall or overwintering withered gall can produce a single D. kuriphilus 
or T. sinensis, respectively. The surface of the D. kuriphilus gall is smooth and intact. All samples were frozen with liquid nitrogen for $30 \mathrm{~min}$ and transported to the laboratory on a dry ice pack, then stored at $-78{ }^{\circ} \mathrm{C}$ until further processing. D. kuriphilus and T. sinensis living in galls were also frozen immediately and stored at $-78{ }^{\circ} \mathrm{C}$. The surfaces of the samples were sterilized with $75 \%$ ethanol for $2 \mathrm{~min}$ and $2.5 \%$ sodium hypochlorite for $5 \mathrm{~min}$, then rinsed with sterile water five times. The D. kuriphilus galls and overwintering withered galls were removed from galled twigs, and then $D$. kuriphilus and T. sinensis were removed from $D$. kuriphilus galls and overwintering withered galls, respectively, with sterile scalpels. The sample size was nine for D. kuriphilus, T. sinensis, D. kuriphilus galls and associated galled twigs group. Each sample of D. kuriphilus and T. sinensis included 10 individuals.

\subsection{DNA Extraction, PCR Amplification and High-Throughput Sequencing}

The DNA extraction of D. kuriphilus female adults, T. sinensis female adults, D. kuriphilus galls and galled twigs was performed with a FastDNA ${ }^{\circledR}$ Spin Kit (MP Biomedicals, Solon, OH, USA). DNA extraction blanks were used with each batch of samples to assess environmental contamination and no amplified PCR products of the DNA extraction blanks were detected.

The V5-V7 region of the bacterial 16S ribosomal RNA was amplified using nested PCR primers, with the first primer pair 799F (5'-AACMGGATTAGATACCCKG-3')-1392R (5'ACGGGCGGTGTGTRC-3') and the second primer pair 799F (5'-AACMGGATTAGATACCCKG$\left.3^{\prime}\right)$-1193R (5'-ACGTCATCCCCACCTTCC-3'). Sterile DNA-free water was used instead of template DNA as the negative control in the $16 \mathrm{~S}$ amplicon screening process to exclude the possibility of amplicon contamination. The PCR was performed on a GeneAmp PCR System 9700 (Applied Biosystems, London, UK) in a $20 \mu \mathrm{L}$ reaction volume: $4 \mu \mathrm{L} 5 \times$ TransStart FastPfu buffer; $0.4 \mu \mathrm{L}$ Taq polymerase; $2 \mu \mathrm{L}$ dNTPs (2.5 mM each); $0.8 \mu \mathrm{L}$ forward and reverse primers $(5 \mu \mathrm{M}) ; 1 \mu \mathrm{L}$ DNA template; and $11 \mu \mathrm{L} \mathrm{H}_{2} \mathrm{O}$. The cycling conditions of the first round of nested PCR were $5 \mathrm{~min}$ at $95^{\circ} \mathrm{C}$, followed by 27 cycles of $30 \mathrm{~s}$ at $95^{\circ} \mathrm{C}, 30 \mathrm{~s}$ at $53{ }^{\circ} \mathrm{C}, 45 \mathrm{~s}$ at $72{ }^{\circ} \mathrm{C}$ and a final elongation step of $15 \mathrm{~min}$ at $72{ }^{\circ} \mathrm{C}$. After the first round of amplification, the second round of amplification was carried out with the same cycling conditions, except that 13 cycles were performed and $1 \mu \mathrm{L}$ of the first round PCR products was used as the template. The PCR products were separated and purified on a $2 \%$ agarose gel and AxyPrep DNA Gel Extraction Kit (Axygen Biosciences, Union City, CA, USA), then quantified with a Quantus ${ }^{\mathrm{TM}}$ Fluorometer (Promega, Madison, WI, USA).

The high-throughput paired-end sequencing was performed on the Illumina MiSeq PE300 sequencing platform (Illumina, San Diego, CA, USA) with a NEXTFLEX Rapid DNA-Seq Kit (Bioo Scientific, Austin, TX, USA) by Majorbio Bio-Pharm Technology Co. Ltd. (Shanghai, China). All raw data have been deposited in the NCBI Sequence Read Archive database under the BioProject accession number PRJNA780913.

\subsection{Bioinformatics and Statistical Analysis}

The original sequences were spliced and quality controlled with FLASH [30] and Trimmomatic [31] software according to the following criteria: sequence length $>200 \mathrm{bp}$; mean quality score $\geq 20$; and no ambiguous bases. Then, the high quality sequences were clustered into Operational Taxonomic Units (OTU) with a 97\% similarity cutoff in Usearch software [32]. According to the Silva database, each high quality sequence was annotated at phylum, class, order, family, genus, species and OTU level using Naive Bayesian classifier [33] and the confidence threshold was 0.8. A total of 31,353 sequences were randomly selected from each sample to generate an OTU table recording the abundance and taxonomy of each OTU.

The OTU tables were imported into R version 3.6.3 (https: / / www.r-project.org, (accessed on 27 October 2021) for subsequent statistical analysis. We counted the number of unique and common bacteria among D. kuriphilus, T. sinensis, D. kuriphilus galls and galled twigs at the species level. Shannon index measures were used to evaluate the $\alpha$-diversity of the bacterial communities in D. kuriphilus, T. sinensis, D. kuriphilus galls and galled 
twigs at the species level. Shapiro-Wilk and Bartlett tests showed that the distribution of the Shannon index was not normal and that the variance of the Shannon index was not homogeneous across the groups. Thus, the nonparametric Kruskal-Wallis test was used to determine whether significant overall differences existed among the Shannon index values of D. kuriphilus, T. sinensis, D. kuriphilus galls and galled twigs, and the Dunn test was used for multiple comparisons when the Kruskal-Wallis test result was significant. Principal coordinate analyses (PCoAs) were carried out to compare the bacterial community structures of D. kuriphilus, T. sinensis, D. kuriphilus galls and galled twigs. First, the overall difference in bacterial community structures was assessed with permutational multivariate analysis of variance (PERMANOVA). PERMANOVA was performed with the "adonis" function in the "vegan" package [34] in R with 1000 permutations according to the weighted UniFrac distance. Second, a PCoA was performed with the "pcoa" function in the R package "ape" according to the weighted UniFrac distance [35].

The linear discriminant analysis (LDA) effect size (LEFSE) (http:/ / huttenhower.sph. harvard.edu/galaxy /, (accessed on 27 October 2021) was used to discover the predominant bacteria associated with $D$. kuriphilus, T. sinensis, D. kuriphilus galls and galled twigs. The Kruskal-Wallis test was used to detect bacterial taxa whose relative abundance significantly differed among D. kuriphilus, T. sinensis, D. kuriphilus galls and galled twigs. Then, LDA was used to calculate the effect size of each taxon; the higher the LDA score, the greater the influence of the taxa on the difference. The LDA score threshold was set to four. The predominant bacteria were those with LDA scores $>4$ and a significantly higher relative abundance than that in other groups.

\section{Results}

3.1. Bacterial Diversity and Community Composition of D. kuriphilus, T. sinensis, D. kuriphilus Galls and Galled Twigs

A total of 14 phyla, 20 classes, 63 orders, 103 families, 181 genera, 273 species and 373 OTUs were identified in the bacterial communities of D. kuriphilus, T. sinensis, D. kuriphilus galls and galled twigs (Table 1). Galled twigs had the most endophytic bacteria, followed by D. kuriphilus galls and T. sinensis, and D. kuriphilus had the fewest bacteria from the phylum to OTU level (Table 1). Similarly, the relative abundance of endophytic bacteria at the species level in galled twigs was the highest, followed by that of bacteria in D. kuriphilus galls, T. sinensis and D. kuriphilus (Figure 1). The changing trend of amount and relative abundance of bacteria could be associated with trophic relationships among D. kuriphilus, T. sinensis, D. kuriphilus galls and galled twigs. The bacterial communities of D. kuriphilus, T. sinensis, D. kuriphilus galls and galled twigs comprised 138, 182, 253 and 266 species, respectively (Table 1 ).

A significant overall difference was observed among the bacterial $\alpha$-diversity (KruskalWallis $\mathrm{H}$ test, $\mathrm{H}_{3,32}=28.34, p<0.01$ ) and community structures (PERMANOVA, $\mathrm{R}^{2}=0.60$, $p<0.01$ ) of D. kuriphilus, T. sinensis, D. kuriphilus galls and galled twigs (Figure 1). The $\alpha$-diversity of the bacterial community in D. kuriphilus was significantly lower than that in T. sinensis, D. kuriphilus galls and galled twigs (Dunn test, $p<0.01$ for all cases), whereas the $\alpha$-diversity of the bacterial community in galled twigs was significantly higher than that in D. kuriphilus, T. sinensis and D. kuriphilus galls at the species level (Dunn test, $p<0.01$ for all cases) (Figure 1). The PCoA indicated that the bacterial community structures of $D$. kuriphilus and T. sinensis clearly differed from those of the other groups (Figure 1). These results suggest that $D$. kuriphilus adults, T. sinensis, D. kuriphilus galls and galled twigs may provide a unique habit for their own bacterial community. 
Table 1. The total number of bacteria in Dryocosmus kuriphilus, Torymus sinensis, D. kuriphilus galls and the galled twigs of Castanea mollissima from phylum to OTU levels.

\begin{tabular}{cccccccc}
\hline & Phylum & Class & Order & Family & Genus & Species & OTU \\
\hline T. sinensis & 10 & 15 & 54 & 80 & 125 & 182 & 221 \\
D. kuriphilus & 8 & 12 & 40 & 64 & 98 & 138 & 162 \\
D. kuriphilus galls & 13 & 19 & 60 & 96 & 170 & 253 & 350 \\
Galled twigs & 14 & 20 & 61 & 100 & 176 & 266 & 366 \\
Total & 14 & 20 & 63 & 103 & 181 & 273 & 373 \\
\hline
\end{tabular}
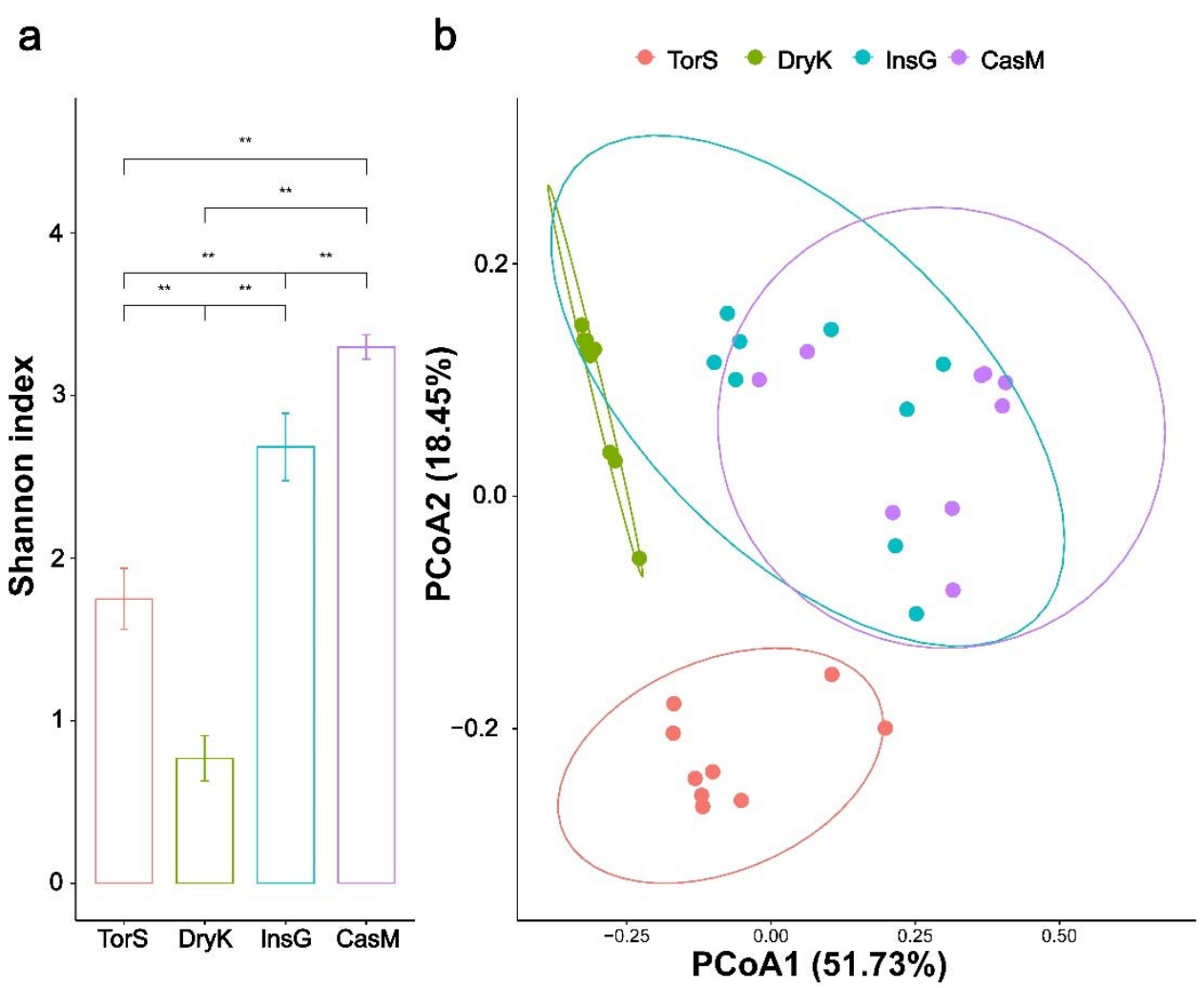

Figure 1. The diversity of bacterial communities in Dryocosmus kuriphilus, Torymus sinensis, D. kuriphilus galls and the galled twigs of Castanea mollissima. (a) The bacterial $\alpha$-diversity of D. kuriphilus, T. sinensis, D. kuriphilus galls and galled twigs at the species level, as measured by the Shannon index. ${ }^{* *}$ indicates a significant difference $(p<0.01)$. (b) The bacterial community structures of D. kuriphilus, T. sinensis, D. kuriphilus galls and the galled twigs based on the weighted UniFrac distance at the species level using Principal coordinates analysis (PCoA). The horizontal and vertical axes indicate the first and second principal coordinates (PCOA1 and PCOA2, respectively). The percentage indicates the proportion of the total variation explained by each principal coordinate. The ellipses represent the $95 \%$ confidence intervals around the centroid for D. kuriphilus, $T$. sinensis, D. kuriphilus galls and the galled twigs. DryK, TorS, InsG and CasM represent D. kuriphilus, T. sinensis, D. kuriphilus galls and the galled twigs of C. mollissima, respectively.

3.2. Unique, Common and Predominant Bacterial Species Associated with D. kuriphilus, T. sinensis, D. kuriphilus Galls and Galled Twigs

A total of 111 bacteria were common to D. kuriphilus adults, T. sinensis, D. kuriphilus galls and galled twigs at the species level (Figure 2). The numbers of unique bacteria in D. kuriphilus, T. sinensis, D. kuriphilus galls and galled twigs were zero, one, one and seven, respectively (Figure 2). These results imply that the bacteria could be transmitted horizontally among D. kuriphilus adults, T. sinensis, D. kuriphilus galls and galled twigs through trophic relationships. 
a

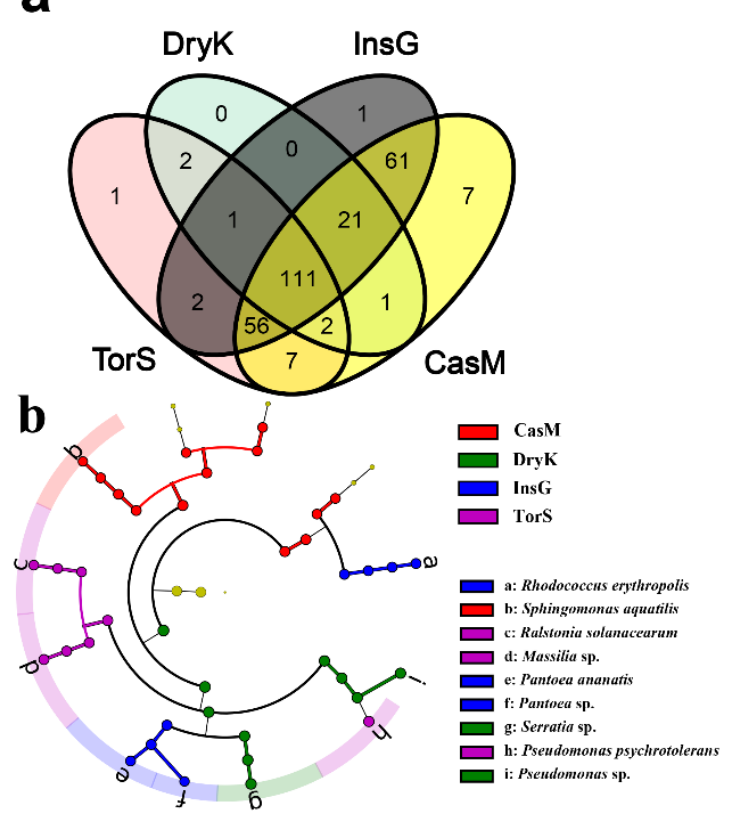

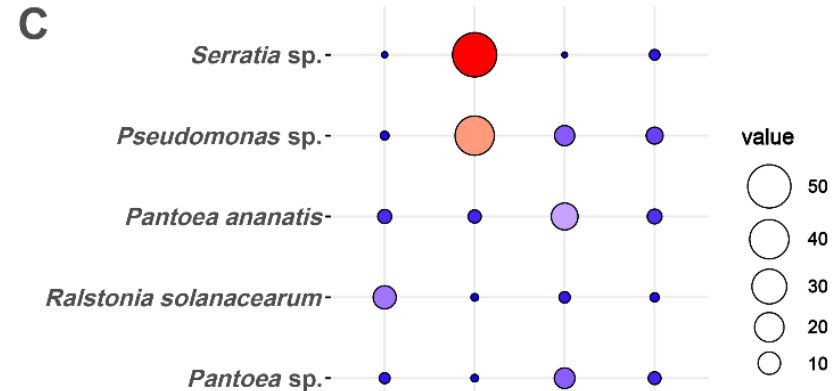

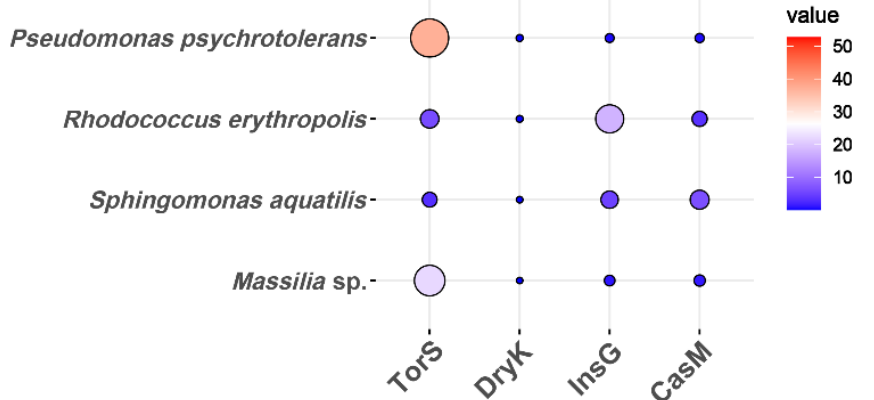

Figure 2. The differences in bacterial community among Dryocosmus kuriphilus, Torymus sinensis, D. kuriphilus galls and the galled twigs of Castanea mollissima. (a) The number of unique and common bacterial species in D. kuriphilus, T. sinensis, D. kuriphilus galls and galled twigs at the species level. The number shows the number of bacterial species unique or common to D. kuriphilus, T. sinensis, D. kuriphilus galls and galled twigs. (b) The LEFSE plot of the predominant bacteria in D. kuriphilus, T. sinensis, D. kuriphilus galls and galled twigs. The cladogram levels, from the inner to outer rings, stand for phylum, class, order, family, genus and species. The green, purple, blue and red nodes of the cladogram show the predominant bacteria in D. kuriphilus, T. sinensis, D. kuriphilus galls and galled twigs from the phylum to species level, respectively. The yellow nodes show the non-dominant bacteria in D. kuriphilus, T. sinensis, D. kuriphilus galls and galled twigs. The letters from a to i show the predominant bacterial species in D. kuriphilus, T. sinensis, D. kuriphilus galls and galled twigs. (c) The bubble chart of the relative abundance of the predominant bacteria in D. kuriphilus, T. sinensis, D. kuriphilus galls and galled twigs at the species level. The area and color of the circles show the relative abundance of the predominant bacteria in D. kuriphilus, T. sinensis, D. kuriphilus galls and galled twigs. The relative abundance is expressed as the percentage of predominant bacteria in the total bacteria. DryK, TorS, InsG and CasM represent D. kuriphilus, T. sinensis, D. kuriphilus galls and galled twigs, respectively.

The LEFSE analysis indicated that a total of two phyla, three classes, seven orders, ten families, eight genera and nine species were predominant in the bacterial communities of D. kuriphilus, T. sinensis, D. kuriphilus galls and galled twigs (Figure 2). The bacterial community of D. kuriphilus was dominated by one phylum, one class, two orders, two families, two genera and two species (Figure 2). The bacterial communities of T. sinensis and $D$. kuriphilus galls were dominated by one order, two families, two genera and three species, whereas the bacterial community of galled twigs was dominated by one phylum, two classes, three orders, four families, two genera and one species (Figure 2).

For D. kuriphilus, the relative abundance of the predominant bacterial species Serratia sp. and Pseudomonas sp. was 52.71\% and 40.1\%, respectively (Figure 2 and Table 2). For T. sinensis, the relative abundance of the predominant bacterial species Pseudomonas psychrotolerans, Massilia sp. and Ralstonia solanacearum was $37.16 \%, 21.98 \%$ and $10.77 \%$, respectively (Figure 2 and Table 2). For D. kuriphilus galls, the relative abundance of the predominant bacterial species Rhodococcus erythropolis, Pantoea ananatis and Pantoea sp. was 17.81\%, $15.93 \%$ and $8.03 \%$, respectively (Figure 2 and Table 2 ). For galled twigs, the relative abun- 
dance of the predominant bacterial species Sphingomonas aquatilis was 6.35\% (Figure 2 and Table 2). These predominant bacteria have never been reported in D. kuriphilus galls and T. sinensis prior to this study (Table 2).

Table 2. A report about the genus of the predominant bacteria in Dryocosmus kuriphilus, Torymus sinensis, D. kuriphilus galls and the galled twigs of Castanea mollissima.

\begin{tabular}{|c|c|c|c|c|}
\hline $\begin{array}{c}\text { Genus of } \\
\text { Predominant Bacteria }\end{array}$ & $\begin{array}{l}\text { Reported in } \\
\text { T. sinensis }\end{array}$ & Reported in Galling Insects & $\begin{array}{l}\text { Reported in } \\
\text { D. kuriphilus galls }\end{array}$ & $\begin{array}{l}\text { Reported in } \\
\text { C. mollissima }\end{array}$ \\
\hline \multicolumn{5}{|l|}{ TorS group } \\
\hline Massilia & No & Leptocybe invasa (Liu et al., 2021) & No & No \\
\hline Ralstonia & No & & No & No \\
\hline Pseudomonas & No & $\begin{array}{l}\text { D. kuriphilus (Iskender et al., 2017), L. invasa } \\
\text { (Liu et al., 2021), Gall midges (Bansal et al., } \\
\text { 2014; Ojha et al., 2017), Gall aphids } \\
\text { (Medina et al., 2011; Wu et al., 2018), Galling } \\
\text { sawflies (Michell and Nyman, 2021), Thrips } \\
\text { (Hammer et al., 2021) }\end{array}$ & No & No \\
\hline \multicolumn{5}{|l|}{ Dryk group } \\
\hline Serratia & No & $\begin{array}{c}\text { Gall wasps (Liu et al., 2021), Gall aphids } \\
\text { (Amit et al., 2017; Medina et al., 2011; } \\
\text { Wu et al., 2018), psyllids } \\
\text { (Morrow et al., 2017) }\end{array}$ & No & Chen et al., 2019 \\
\hline Pseudomonas & No & $\begin{array}{l}\text { D. kuriphilus (Iskender et al., 2017), L. invasa } \\
\text { (Liu et al., 2021), Gall midges (Bansal et al., } \\
\text { 2014; Ojha et al., 2017), Gall aphids (Medina } \\
\text { et al., 2011; Wu et al., 2018), Galling sawflies } \\
\text { (Michell and Nyman, 2021), Thrips } \\
\text { (Hammer et al., 2021) }\end{array}$ & No & Ni, 1998; \\
\hline InsG group & & & No & \\
\hline Pantoea & No & $\begin{array}{c}\text { L. invasa (Liu et al., 2021), Gall midges } \\
\text { (Bansal et al., 2014; Ojha et al., 2017), Thrips } \\
\text { (Hammer et al., 2021) }\end{array}$ & No & Zhang et al., 2019 \\
\hline Rhodococcus & No & Gall aphids (Wu et al., 2018) & No & No \\
\hline \multicolumn{5}{|l|}{ CasM group } \\
\hline Sphingomonas & No & $\begin{array}{l}\text { L. invasa (Guo et al., 2020), Galling sawflies } \\
\text { (Michell and Nyman, 2021), Gall aphids } \\
\text { (Wu et al., 2018) }\end{array}$ & No & Chen et al., 2019 \\
\hline
\end{tabular}

\section{Discussion}

4.1. Possibility of Horizontal Transmission of Bacteria among D. kuriphilus, T. sinensis, D. kuriphilus Galls and Galled Twigs

D. kuriphilus, T. sinensis, D. kuriphilus galls and galled twigs shared most bacteria at the species level, thus suggesting that the bacteria might be transmitted horizontally among D. kuriphilus adults, T. sinensis adults, D. kuriphilus galls and galled twigs. We speculated that ecological interactions might be associated with the potential horizontal transmission among D. kuriphilus, T. sinensis, D. kuriphilus galls and galled twigs.

The water and most of the nutrients of D. kuriphilus galls are obtained from the host plants via the vascular system [8], and the transportation of these substances may provide favorable conditions for the horizontal transmission of bacteria. For example, previous studies have confirmed that the species of the Serratia and Pseudomonas genera can migrate from root to leaf via the transportation of substances through the vascular system, according to green fluorescent protein labeling and $\beta$-glucuronidase staining [36,37]. Furthermore, for the D. kuriphilus adults, there is a subset of bacteria from D. kuriphilus galls. 
D. kuriphilus galls are the sole food source for D. kuriphilus, and plant endophytes can enter the digestive system of phytophagous insects through feeding [38-40]. Thus, the feeding relationship between $D$. kuriphilus and $D$. kuriphilus galls may be beneficial for the horizontal transmission of bacteria, and most bacteria of $D$. kuriphilus may be derived from $D$. kuriphilus galls. T. sinensis is an important natural enemy of D. kuriphilus, and feeds on the D. kuriphilus larvae. Our studies suggest the horizontal transmission of Wolbachia between the gall wasp Andricus mukaigawae and parasitoids Torymus sp. [40]. Previous studies have confirmed the horizontal transmission of bacteria between insect hosts and three parasitoids using florescence in situ hybridization and transmission electron microscopy [41]. We suggest that the parasitic relationship between $T$. sinensis and D. kuriphilus may provide a potential route for the horizontal transmission of bacteria. Furthermore, some species of Torymus can feed on plant material [12] and T. sinensis may acquire the bacteria from overwintering withered galls.

\subsection{Differences in Bacterial Community Structures among D. kuriphilus, T. sinensis, D. kuriphilus} Galls and Galled Twigs

The chemical characteristics of $D$. kuriphilus galls may be associated with the differences in bacterial community structures between D. kuriphilus galls and galled twigs. First, the concentrations of auxins and cytokinins in galled twigs are different from those in galls, such as midge [42], aphid [43], fly [44], psyllid [45,46], leafhopper [47], sawfly [48], L. invasa [49] and D. kuriphilus galls [50]. The auxins and cytokinins can directly or indirectly affect the physiology of bacteria. For example, the auxins and cytokinins can directly affect the stress-related genes of bacteria as important signaling molecules [51]. Moreover, auxins and cytokinins can indirectly affect bacteria through communicating with jasmonic acid and salicylic acid signaling pathways, which are two major pathways involved in the plant's defense against attacks by pathogens [52,53]. Second, the concentrations of amino acids, carbohydrates, lipids, lignin and secondary metabolites in cynipid galls markedly differ from those in ungalled host plant tissue [8,54-58]. The nutrients in cynipid galls provide unique carbon and nitrogen sources for the bacterial community, and high levels of tannin, polyphenol oxidase and reactive oxygen species in cynipid galls can inhibit the growth of some bacteria $[54,59,60]$. Thus, we suggest that the compositions and concentrations of chemical substances in D. kuriphilus galls provide a special habitat for the bacteria associated with D. kuriphilus galls.

The differences in bacterial community composition between $D$. kuriphilus and T. sinensis may be associated with the life history of D. kuriphilus and T. sinensis. First, D. kuriphilus is an herbivorous insect and T. sinensis is a parasitoid [61]. The differences in food sources may affect the differences in bacterial community composition between $D$. kuriphilus and $T$. sinensis. Second, the species barriers between $D$. kuriphilus and T. sinensis may prevent the colonization of bacteria [12,62]. Third, T. sinensis adults emerge from the withered overwinter galls whereas D. kuriphilus adults emerge from newly formed galls [9]. The overwintering withered galls were induced by D. kuriphilus in the previous year, and the environment of $D$. kuriphilus is different from that of T. sinensis. Thus, we suggest that the food sources, species barriers and gall environment may affect the differences in bacterial communities between D. kuriphilus and T. sinensis.

\subsection{Predominant Bacteria Associated with D. kuriphilus}

The Serratia sp. and Pseudomonas sp. are predominant in D. kuriphilus and widely distributed in galling insects, including D. kuriphilus, gall midges, gall aphids, gall sawflies and thrips $[17,18,23-27,63,64]$. Many species of the Serratia and Pseudomonas genera are plant pathogenic bacteria [65]. D. kuriphilus lay eggs in the buds of host plants through its ovipositor, and the buds come into contact with the ovipositor and ovipositional fluid [66]. Furthermore, D. kuriphilus galls come into contact with the feces and saliva of D. kuriphilus [67]. We speculate that the Serratia sp. and Pseudomonas sp. of D. kuriphilus may be plant pathogenic bacteria, and D. kuriphilus adults may be potential vectors of these plant pathogens. 
Moreover, some species of the Pseudomonas genus can synthetize auxins and cytokinins, both of which have been identified in various galling insects, including gall midges [42], gall aphids [43], fruit flies [44], leafhoppers [47], gall sawflies [48] and psyllids [45,46]. Recent studies have suggested that the fast growth of gall induction may not be consistently mediated by a bacterial symbiont [27]. However, it remains unclear whether the bacteria are associated with the auxins and cytokinins in galling insects. In future studies, we plan to culture the Pseudomonas sp. in D. kuriphilus and explore its potential roles.

\section{Conclusions}

In conclusion, we provide the first evidence that D. kuriphilus shares most bacterial species with T. sinensis, D. kuriphilus galls and galled twigs, and are dominated by Serratia sp. and Pseudomonas sp. Furthermore, the bacterial community structures of D. kuriphilus and T. sinensis clearly differ from those of the other groups.

We suggest that vascular transportation, feeding and parasitic relationships may be associated with horizontal transmission among D. kuriphilus, T. sinensis, D. kuriphilus galls and galled twigs. Furthermore, the chemical characteristics of $D$. kuriphilus galls may be associated with the differences in bacterial community structures between $D$. kuriphilus galls and galled twigs. The food sources, species barriers and gall environment may affect the differences in bacterial communities between D. kuriphilus and T. sinensis. In addition, we suggest that $D$. kuriphilus may be a potential vector of plant pathogens.

Supplementary Materials: The following are available online at https:/ /www.mdpi.com/article/10 .3390/insects13010086/s1: Figure S1, the galls induced by Dryocosmus kuriphilus and galled twigs.

Author Contributions: Conceptualization, X.Y. (Xiaohui Yang) and D.Z.; methodology, X.Y. (Xiaohui Yang) and D.Z.; formal analysis: X.Y. (Xiaohui Yang), Y.H. and D.Z.; investigation, X.Y. (Xiaohui Yang) and Y.H.; writing — original draft preparation, X.Y. (Xiaohui Yang), Y.H. and D.Z.; writing-review and editing, X.Y. (Xiaohui Yang), Y.H., D.Z., Y.Z., L.Z., X.Y. (Xuemei Yang) and Y.W.; visualization, X.Y. (Xiaohui Yang); supervision, X.Y. (Xiaohui Yang) and D.Z.; funding acquisition, X.Y. (Xiaohui Yang). All authors have read and agreed to the published version of the manuscript.

Funding: This research was funded by the National Key Research and Development Program of China (grant number 2018YFE0127100) and the National Natural Science Foundation of China (grant number 31400550).

Institutional Review Board Statement: Not applicable.

Informed Consent Statement: Not applicable.

Data Availability Statement: The raw data were deposited into the NCBI Sequence Read Archive (SRA) database under BioProject accession number PRJNA780913.

Acknowledgments: We thank Zhiwei Liu of Eastern Illinois University for the identification of Dryocosmus kuriphilus and Torymus sinensis. The host plant Castanea mollissima was kindly identified by Xun-Lin Yu of the Central South University of Forestry and Technology.

Conflicts of Interest: The authors have no conflict of interest to declare.

\section{References}

1. Dodueva, I.E.; Lebedeva, M.A.; Kuznetsova, K.A.; Gancheva, M.S.; Paponova, S.S.; Lutova, L.L. Plant tumors: A hundred years of study. Planta 2020, 251, 82. [CrossRef]

2. Harris, M.O.; Pitzschke, A. Plants make galls to accommodate foreigners: Some are friends, most are foes. New Phytol. 2020, 225, 1852-1872. [CrossRef]

3. Egan, S.P.; Hood, G.R.; Martinson, E.O.; Ott, J.R. Cynipid gall wasps. Curr. Biol. 2018, 28, R1370-R1374. [CrossRef]

4. Avtzis, D.N.; Melika, G.; Matošević, D.; Coyle, D.R. The Asian chestnut gall wasp Dryocosmus kuriphilus: A global invader and a successful case of classical biological control. J. Pest. Sci. 2019, 92, 107-115. [CrossRef]

5. Gehring, E.; Bellosi, B.; Reynaud, N.; Conedera, M. Chestnut tree damage evolution due to Dryocosmus kuriphilus attacks. J. Pest. Sci. 2020, 93, 103-115. [CrossRef] 
6. Bernardo, U.; Iodice, L.; Sasso, R.; Tutore, V.A.; Cascone, P.; Guerrieri, E. Biology and monitoring of Dryocosmus kuriphilus on Castanea sativa in southern Italy. Agric. For. Entomol. 2013, 15, 65-76. [CrossRef]

7. Cooper, W.R.; Rieske, L.K. Community associates of an exotic gallmaker, Dryocosmus kuriphilus (Hymenoptera: Cynipidae), in eastern north America. Ann. Entomol. Soc. Am. 2007, 100, 236-244. [CrossRef]

8. Reale, L.; Tedeschini, E.; Rondoni, G.; Ricci, C.; Bin, F.; Frenguelli, G.; Ferranti, F. Histological investigation on gall development induced by a worldwide invasive pest, Dryocosmus kuriphilus, on Castanea sativa. Plant Biosyst. 2016, 150, 35-42. [CrossRef]

9. Matosevic, D.; Lackovic, N.; Melika, G.; Kos, K.; Franic, I.; Kriston, E.; Bozso, M.; Seljak, G.; Rot, M. Biological control of invasive Dryocosmus kuriphilus with introduced parasitoid Torymus sinensis in Croatia, Slovenia and Hungary. Period. Biol. 2016, 117, 471-477. [CrossRef]

10. Aebi, A.; Schönrogge, K.; Melika, G.; Alma, A.; Bosio, G.; Quacchia, A.; Picciau, L.; Abe, Y.; Moriya, S.; Yara, K. Parasitoid recruitment to the globally invasive chestnut gall wasp Dryocosmus kuriphilus. In Galling Arthropods and Their Associates; Ozaki, K., Ed.; Springer: Tokyo, Japan, 2006; pp. 103-121.

11. Graziosi, I.; Rieske, L.K. Response of Torymus sinensis, a parasitoid of the gallforming Dryocosmus kuriphilus, to olfactory and visual cues. Biol. Control 2013, 67, 137-142. [CrossRef]

12. Quacchia, A.; Moriya, S.; Bosio, G.; Radócz, L.; Botu, M.; Bolvanský, M. Effectiveness of Torymus sinensis in the biological control of Dryocosmus kuriphilus in Italy. Acta Hortic. 2014, 199-204. [CrossRef]

13. Yara, K. Interaction between Torymus sinensis (Hymenoptera: Torymidae) and T. beneficus, introduced and indigenous parasitoids of the chestnut gall wasp Dryocosmus kuriphilus (Hymenoptera: Cynipidae). Jarq-Jpn. Agr. Res. Q. 2014, 48, 35-40. [CrossRef]

14. Hayward, A.; Stone, G.N. Oak gall wasp communities: Evolution and ecology. Basic Appl. Ecol. 2005, 6, 435-443. [CrossRef]

15. Hansen, A.K.; Moran, N.A. The impact of microbial symbionts on host plant utilization by herbivorous insects. Mol. Ecol. 2014, 23, 1473-1496. [CrossRef]

16. Guo, C.; Peng, X.; Zheng, X.; Wang, X.; Wang, R.; Huang, Z.; Yang, Z. Comparison of bacterial diversity and abundance between sexes of Leptocybe invasa Fisher \& La Salle (Hymenoptera: Eulophidae) from China. PeerJ 2020, 8, e8411.

17. Liu, Y.; Xu, L.; Zhang, Z.; Huang, Z.; Fang, D.; Zheng, X.; Yang, Z.; Lu, M. Isolation, identification, and analysis of potential functions of culturable bacteria associated with an invasive gall wasp. Leptocybe invasa. Microb. Ecol. 2021, 1-16. [CrossRef]

18. Wu, H.; Chen, X.; Hang, C.; Qin, L.; Chen, M.S. Variation and diversification of the microbiome of Schlechtendalia chinensis on two alternate host plants. PLoS ONE 2018, 13, e0200049. [CrossRef]

19. Yates, A.D.; Michel, A. Mechanisms of aphid adaptation to host plant resistance. Curr. Opin. Insect Sci. 2018, 26, 41-49. [CrossRef]

20. Correa, C.C.; Ballard, J.W.O. Wolbachia associations with insects: Winning or losing against a master manipulator. Front. Ecol. Evol. 2016, 3, 153. [CrossRef]

21. Zhu, D.H.; He, Y.Y.; Fan, Y.S.; Ma, M.Y.; Peng, D.L. Negative evidence of parthenogenesis induction by Wolbachia in a gallwasp species, Dryocosmus kuriphilus. Entomol. Exp. Appl. 2007, 124, 279-284. [CrossRef]

22. Iskender, N.A.; Algur, O.F.; Aksu, Y.; Saral, A. Isolation, identification and characterization of biotechnologically important bacteria from microflora of Dryocosmus kuriphilus Yasumatsu (Hymenoptera: Cynipidae). Biotechnol. Biotechnol. Equip. 2017, 31, 505-510. [CrossRef]

23. Bansal, R.; Hulbert, S.H.; Reese, J.C.; Whitworth, R.J.; Stuart, J.J.; Chen, M.S. Pyrosequencing reveals the predominance of pseudomonadaceae in gut microbiome of a gall midge. Pathogens 2014, 3, 459-472. [CrossRef]

24. Ojha, A.; Sinha, D.K.; Padmakumari, A.P.; Bentur, J.S.; Nair, S. Bacterial community structure in the Asian rice gall midge reveals a varied microbiome rich in Proteobacteria. Sci. Rep. 2017, 7, 1-13.

25. Medina, R.F.; Nachappa, P.; Tamborindeguy, C. Differences in bacterial diversity of host-associated populations of Phylloxera notabilis Pergande (Hemiptera: Phylloxeridae) in pecan and water hickory. J. Evol. Biol. 2011, 24, 761-771. [CrossRef] [PubMed]

26. Michell, C.T.; Nyman, T. Microbiomes of willow-galling sawflies: Effects of host plant, gall type, and phylogeny on community structure and function. Genome 2021, 64, 615-626. [CrossRef] [PubMed]

27. Hammer, T.J.; Clerck-Floate, R.D.; Tooker, J.F.; Price, P.W.; Miller, D.G.; Connor, E.F. Are bacterial symbionts associated with gall induction in insects? Arthropod-Plant Inte. 2021, 15, 1-12. [CrossRef]

28. Hou, H.Q.; Zhao, G.Z.; Su, C.Y.; Zhu, D.H. Wolbachia prevalence patterns: Horizontal transmission, recombination, and multiple infections in chestnut gall wasp-parasitoid communities. Entomol. Exp. Appl. 2020, 168, 752-765. [CrossRef]

29. Yang, X.-M.; Hui, Y.; Zhao, L.-Q.; Zhu, D.-H.; Zeng, Y.; Yang, X.-H. Comparison of auxin and cytokinins concentrations, and the structure of bacterial community between host twigs and Lithosaphonecrus arcoverticus galls. Insects 2021, 12, 982. [CrossRef]

30. Magoc, T.; Salzberg, S.L. Flash: Fast length adjustment of short reads to improve genome assemblies. Bioinformatics 2011, 27, 2957-2963. [CrossRef] [PubMed]

31. Bolger, A.M.; Marc, L.; Bjoern, U. Trimmomatic: A flexible trimmer for Illumina sequence data. Bioinformatics 2014, 30, 2114-2120. [CrossRef]

32. Edgar, R.C. Search and clustering orders of magnitude faster than BLAST. Bioinformatics 2010, 26, 2460-2461. [CrossRef]

33. Wang, Q.; Garrity, G.M.; Tiedje, J.M.; Cole, J.R. Naive Bayesian classifier for rapid assignment of rRNA sequences into the new bacterial taxonomy. Appl. Environ. Microb. 2007, 73, 5261-5267. [CrossRef]

34. Oksanen, J.; Blanchet, F.G.; Kindt, R.; Legendre, P.; Minchin, P.R.; O’hara, R.; Simpson, G.L.; Solymos, P.; Stevens, M.H.H.; Wagner, H.; et al. Package 'vegan'. Community Ecol. Package 2013, 2, 1-295. 
35. Paradis, E.; Schliep, K. Ape 5.0: An environment for modern phylogenetics and evolutionary analyses in R. Bioinformatics 2019, 35, 526-528. [CrossRef]

36. Kandel, S.L.; Joubert, P.M.; Doty, S.L. Bacterial endophyte colonization and distribution within plants. Microorganisms 2017, 5, 77. [CrossRef]

37. Johnston-Monje, D.; Raizada, M.N. Conservation and diversity of seed associated endophytes in Zea across boundaries of evolution, ethnography and ecology. PLoS ONE 2011, 6, e20396. [CrossRef]

38. Jang, S.; Kikuchi, Y. Impact of the insect gut microbiota on ecology, evolution, and industry. Curr. Opin. Insect Sci. 2020, 41, 33-39. [CrossRef]

39. Janson, E.M.; Stireman, J.O., III; Singer, M.S.; Abbot, P. Phytophagous insect-microbe mutualisms and adaptive evolutionary diversification. Evolution 2008, 62, 997-1012. [CrossRef]

40. Yang, X.H.; Zhu, D.H.; Liu, Z.; Zhao, L.; Su, C.Y. High levels of multiple infections, recombination and horizontal transmission of Wolbachia in the Andricus mukaigawae (Hymenoptera; Cynipidae) communities. PLoS ONE 2013, 8, e78970. [CrossRef]

41. Chiel, E.; Zchori-Fein, E.; Inbar, M.; Gottlieb, Y.; Adachi-Hagimori, T.; Kelly, S.E.; Asplen, M.K.; Hunter, M.S. Almost there: Transmission routes of bacterial symbionts between trophic levels. PLoS ONE 2009, 4, e4767. [CrossRef]

42. Tanaka, Y.; Okada, K.; Asami, T.; Suzuki, Y. Phytohormones in Japanese mugwort gall induction by a gall-inducing gall midge Biosci. Biotech. Bioch 2013, 77, 1942-1948. [CrossRef]

43. Takei, M.; Yoshida, S.; Kawai, T.; Hasegawa, M.; Suzuki, Y. Adaptive significance of gall formation for a gall-inducing aphids on Japanese elm trees. J. Insect Physiol. 2015, 72, 43-51. [CrossRef]

44. Mapes, C.C.; Davies, P.J. Indole-3-acetic acid and ball gall development on Solidago altissima. New Phytol. 2001, 151, 195-202. [CrossRef]

45. Straka, J.R.; Hayward, A.R.; Emery, R.N. Gall-inducing Pachypsylla celtidis (Psyllidae) infiltrate hackberry trees with high concentrations of phytohormones. J. Plant Interact. 2010, 5, 197-203. [CrossRef]

46. Kai, S.; Kumashiro, S.; Adachi, S.; Suzuki, Y.; Shiomi, Y.; Matsunaga, K.; Gyoutoku, N.; Asami, T.; Tokuda, M. Life history of Stenopsylla nigricornis (Hemiptera: Psylloidea: Triozidae) and phytohormones involved in its gall induction. Arthropod-Plant Inte. 2017, 11, 99-108. [CrossRef]

47. Tokuda, M.; Jikumaru, Y.; Matsukura, K.; Takebayashi, Y.; Kumashiro, S.; Matsumura, M.; Kamiya, Y. Phytohormones related to host plant manipulation by a gall-inducing leafhopper. PLoS ONE 2013, 8, e62350. [CrossRef]

48. Yamaguchi, H.; Tanaka, H.; Hasegawa, M.; Tokuda, M.; Asami, T.; Suzuki, Y. Phytohormones and willow gall induction by a gall-inducing sawfly. New Phytol. 2012, 196, 586-595. [CrossRef]

49. Li, X.Q.; Liu, Y.Z.; Guo, W.F.; Solanki, M.K.; Yang, Z.D.; Xiang, Y.; Ma, Z.C.; Wen, Y.G. The gall wasp Leptocybe invasa (Hymenoptera: Eulophidae) stimulates different chemical and phytohormone responses in two Eucalyptus varieties that vary in susceptibility to galling. Tree Physiol. 2017, 37, 1208-1217. [CrossRef]

50. Wood, B.W.; Payne, J.A. Growth regulators in chestnut shoot galls infected with oriental ghestnut gall wasp, Dryocosmus kuriphilus (Hymenoptera: Cynipidae). Environ. Entomol. 1988, 17, 915-920. [CrossRef]

51. Donati, A.J.; Lee, H.-I.; Leveau, J.H.J.; Chang, W.-S. Effects of indole-3-acetic acid on the transcriptional activities and stress tolerance of Bradyrhizobium japonicum. PLoS ONE 2013, 8, e76559. [CrossRef]

52. Yang, J.; Duan, G.; Li, C.; Liu, L.; Han, G.; Zhang, Y.; Wang, C. The crosstalks between jasmonic acid and other plant hormone signaling highlight the involvement of jasmonic acid as a core component in plant response to biotic and abiotic stresses. Front. Plant Sci. 2019, 10, 1349. [CrossRef]

53. Berens, M.L.; Berry, H.M.; Mine, A.; Argueso, C.T.; Tsuda, K. Evolution of hormone signaling networks in plant defense. Annu. Rev. Phytopathol. 2017, 55, 401-425. [CrossRef]

54. Ikai, N.; Hijii, N. Manipulation of tannins in oaks by galling cynipids. J. Forest Res. 2007, 12, 316-319. [CrossRef]

55. Koncz, N.K.; Szabó, L.J.; Máthé, C.; Jámbrik, K.; M-Hamvas, M. Histological study of quercus galls of Neuroterus quercusbaccarum (Linnaeus, 1758) (Hymenoptera: Cynipidae). Acta Bio. Szeg. 2011, 55, 247-253.

56. Allison, S.D.; Schultz, J.C. Biochemical responses of chestnut oak to a galling cynipid. J. Chem. Ecol. 2005, 31, 151-166. [CrossRef]

57. Li, X.-M.; Yang, X.-H. Comparison of the contents or activities of nutritional and defensive substances between the larval galls and host plants of Dryocosmus kuriphilus. Life Sci. Res. 2019, 23, 214-218.

58. Yang, X.-H.; Li, X.-M.; Zhu, D.-H. Alteration of free amino acid concentrations in insect galls induced by Andricus mukaigawae (Hymenoptera; Cynipidae). Ecol. Entomol. 2020, 45, 945-954. [CrossRef]

59. Chen, H.; Liu, J.; Cui, K.; Lu, Q.; Wang, C.; Wu, H.X.; Yang, Z.X.; Ding, W.F.; Shao, S.X.; Wang, H.Y.; et al. Molecular mechanisms of tannin accumulation in Rhus galls and genes involved in plant-insect interactions. Sci. Rep. 2018, 8, 1-12. [CrossRef]

60. Barbehenn, R.V.; Peter Constabel, C. Tannins in plant-herbivore interactions. Phytochemistry 2011, 72, 1551-1565. [CrossRef]

61. Ferracini, C.; Ferrari, E.; Pontini, M.; Nova, L.K.H.; Saladini, M.A.; Alma, A. Post-release evaluation of non-target effects of Torymus sinensis, the biological control agent of Dryocosmus kuriphilus in Italy. BioControl 2017, 62, 445-456. [CrossRef]

62. Gibbs, M.; Schonrogge, K.; Alma, A.; Melika, G.; Quacchia, A.; Stone, G.N.; Aebi, A. Torymus sinensis: A viable management option for the biological control of Dryocosmus kuriphilus in Europe? BioControl 2011, 56, 527-538. [CrossRef]

63. Amit, L.; Ben-Shlomo, R.; Chiel, E. Are microbial symbionts involved in the speciation of the gall-inducing aphid Slavum wertheimae? Arthropod-Plant Int. 2017, 11, 475-484. [CrossRef]

64. Morrow, J.L.; Hall, A.; Riegler, M. Symbionts in waiting: The dynamics of incipient endosymbiont complementation and replacement in minimal bacterial communities of psyllids. Microbiome 2017, 5, 1-23. [CrossRef] 
65. Van Baarlen, P.; Van Belkum, A.; Summerbell, R.C.; Crous, P.W.; Thomma, B.P.H.J. Molecular mechanisms of pathogenicity: How do pathogenic microorganisms develop cross-kingdom host jumps? FEMS Microbiol. Rev. 2007, 31, 239-277. [CrossRef]

66. Martinez-Sañudo, I.; Mazzon, L.; Simonato, M.; Avtzis, D.; Pujade-Villar, J.; Faccoli, M. Tracking the origin and dispersal of the Asian chestnut gall wasp Dryocosmus kuriphilus Yasumatsu (Hymenoptera, Cynipidae) in Europe with molecular markers. Bull. Entomol. Res. 2019, 109, 300-308. [CrossRef] [PubMed]

67. Stone, G.N.; Schönrogge, K.; Atkinson, R.J.; Bellido, D.; Pujade-Villar, J. The population biology of oak gall wasps (Hymenoptera: Cynipidae). Annu. Rev. Entomol. 2002, 47, 633-668. [CrossRef] 